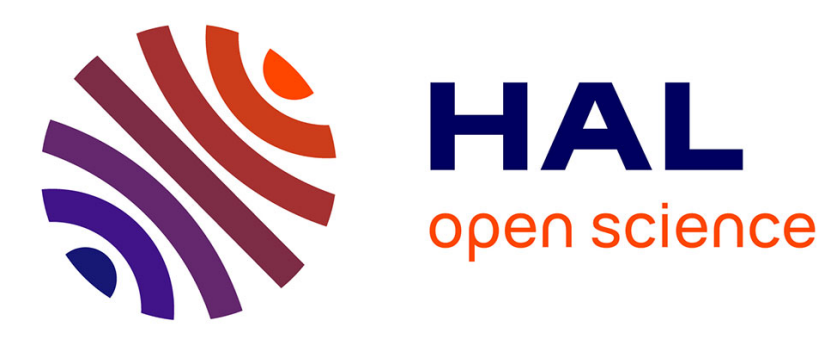

\title{
Réchauffement climatique : les perspectives des rapports entre science, politique et société
}

\author{
Jean Jouzel, Valérie Masson-Delmotte
}

\section{To cite this version:}

Jean Jouzel, Valérie Masson-Delmotte. Réchauffement climatique : les perspectives des rapports entre science, politique et société. Annales des mines - Série Responsabilité et environnement, 2016, $\mathrm{N}^{\circ} 83$ (3), pp.50-54. 10.3917/re1.083.0050 . hal-03335710

\section{HAL Id: hal-03335710 https://hal.science/hal-03335710}

Submitted on 6 Sep 2021

HAL is a multi-disciplinary open access archive for the deposit and dissemination of scientific research documents, whether they are published or not. The documents may come from teaching and research institutions in France or abroad, or from public or private research centers.
L'archive ouverte pluridisciplinaire HAL, est destinée au dépôt et à la diffusion de documents scientifiques de niveau recherche, publiés ou non, émanant des établissements d'enseignement et de recherche français ou étrangers, des laboratoires publics ou privés. 


\title{
RÉCHAUFFEMENT CLIMATIQUE : LES PERSPECTIVES DES RAPPORTS ENTRE SCIENCE, POLITIQUE ET SOCIÉTÉ
}

\author{
Jean Jouzel, Valérie Masson-Delmotte
}

F.F.E. | «Annales des Mines - Responsabilité et environnement »

2016/3 Nº 83 | pages 50 à 54

ISSN $1268-4783$

Article disponible en ligne à l'adresse :

https://www.cairn.info/revue-responsabilite-et-environnement-2016-3-page-50.htm

Distribution électronique Cairn.info pour F.F.E..

(C) F.F.E.. Tous droits réservés pour tous pays.

La reproduction ou représentation de cet article, notamment par photocopie, n'est autorisée que dans les limites des conditions générales d'utilisation du site ou, le cas échéant, des conditions générales de la licence souscrite par votre établissement. Toute autre reproduction ou représentation, en tout ou partie, sous quelque forme et de quelque manière que ce soit, est interdite sauf accord préalable et écrit de l'éditeur, en dehors des cas prévus par la législation en vigueur en France. Il est précisé que son stockage dans une base de données est également interdit. 

les perspectives des rapports entre science, politique et société

\author{
Par Jean JOUZEL et Valérie MASSON-DELMOTTE \\ Laboratoire des sciences du climat et de l'environnement, Institut Pierre-Simon Laplace, CEA-CNRS- \\ UVSQ, Université Paris Saclay
}

\begin{abstract}
Le 22 avril 2016, 175 pays et l'Union européenne ont signé au siège des Nations Unies, à New York, l'Accord de Paris sur le climat. La vingtaine de pays absents à ce rendez-vous ont jusqu'au 21 avril 2017 pour y apposer leur signature. II s'agit du premier accord universel sur le climat. Malgré le fossé entre les contributions annoncées par ces pays et l'objectif affiché d'une limitation de l'élévation de la température moyenne de la Planète bien en-dessous de $2^{\circ} \mathrm{C}$, voire en-dessous de $1,5^{\circ} \mathrm{C}$ par rapport au niveau préindustriel, ce caractère universel justifie pleinement que la COP 21 soit considérée comme un succès. Cette réussite a été possible grâce à l'engagement et aux efforts conjugués d'une communauté scientifique quasi unanime dans son diagnostic, de décideurs politiques qui ont compris l'importance des enjeux et de nombreuses composantes de ce qu'il est convenu d'appeler la société civile. Nous analysons dans cet article la façon dont cette synergie s'est progressivement mise en place.
\end{abstract}

\section{L}

'Accord de Paris est décrit comme ambitieux, équilibré, dynamique et juridiquement contraignant. parti de la transparence : chaque pays a mis sur la table ses objectifs de réduction d'émissions de gaz à effet de serre entre 2020 et $2025-2030$ et ses efforts d'adaptation au changement climatique. Tous les cinq ans, ces objectifs pourront être révisés à la hausse sur la base d'un inventaire global et en s'appuyant sur les meilleures connaissances scientifiques, en particulier sur les rapports d'évaluation du Groupe intergouvernemental d'experts sur l'évolution du climat (GIEC).

Même si tous les engagements étaient réalisés en l'état, les émissions de 2030 seraient de 35 à $40 \%$ trop élevées par rapport aux trajectoires compatibles avec l'objectif de stabilisation du réchauffement atmosphérique à $2^{\circ} \mathrm{C}$. Malgré ce fossé, l'Accord de Paris est à juste titre considéré comme un succès, en premier lieu de par son caractère universel. Ce succès s'est construit par étapes successives : celle du diagnostic des scientifiques a été suivie de la prise de conscience des décideurs politiques et, plus récemment, d'une implication croissante de certaines des composantes de la société civile, qu'il est cependant nécessaire d'élargir si l'on veut que l'objectif des $2^{\circ} \mathrm{C}$ puisse être respecté.

Après des travaux pionniers menés au XIX'e siècle sur l'effet de serre, comme ceux de Joseph Fourier ou d'Arrhénius, l'action potentielle des activités humaines sur le climat a émergé en tant qu'enjeu scientifique majeur au cours des années 1970 suite au constat d'une augmentation de la concentration en gaz à effet de serre dans l'atmosphère. Les premières simulations numériques du climat indiquent qu'un doublement de la teneur en dioxyde de carbone $\left(\mathrm{CO}_{2}\right)$ de l'atmosphère se traduirait par un réchauffement à la surface de la Terre compris entre 1,5 et $4,5^{\circ} \mathrm{C}$ (suivant le modèle considéré).

Une large partie de la communauté scientifique se mobilise pour analyser toutes les facettes des interférences entre les activités humaines et le système climatique. Les résultats publiés en 1987 à partir de l'analyse des glaces antarctiques de Vostok participent à cette prise de conscience : au cours des 150000 dernières années, les variations du climat et de la concentration atmosphérique en $\mathrm{CO}_{2}$ ont été étroitement liées. De plus, l'augmentation récente de la concentration atmosphérique de ce composé due aux activités humaines (utilisation de combustibles fossiles et déforestation) marque une rupture par rapport à la gamme de ses variations naturelles. Ces résultats seront confirmés par des forages antarctiques successifs, qui permettent désormais de connaître ces variations naturelles au cours des 800 millénaires écoulés. La communauté scientifique internationale s'organise rapidement. Ainsi, dès 1979, l'Organisation météorologique mondiale (OMM) a mis sur les rails le Programme de recherche 
mondial sur le climat, suivi par d'autres initiatives qui ont permis d'amplifier les recherches sur l'observation du système climatique, la compréhension des processus en jeu et, bien sûr, l'évolution passée et future de notre climat.

Les politiques ne tardent pas à emboîter le pas. Les scientifiques les ont rapidement convaincus de la nécessité d'établir un diagnostic sur l'impact potentiel des activités humaines sur le climat. En 1988, à l'initiative du G7 - dont sont alors membres Margaret Thatcher et George Bush -, est créé le GIEC, qui est placé sous les auspices conjoints de deux organisations dépendant de I'ONU, le Programme des Nations Unies pour l'environnement et l'Organisation météorologique mondiale (OMM).

La mise en place du GIEC doit aussi beaucoup à des scientifiques de renom, parmi lesquels le Suédois Bert Bolin, qui en sera le premier président ${ }^{(1)}$. Publié en 1990, le premier rapport du GIEC confirme que les activités humaines sont à l'origine d'une augmentation de l'effet de serre à travers l'accroissement des concentrations atmosphériques de $\mathrm{CO}_{2}$ et d'autres gaz à effet de serre, tels que le méthane $\left(\mathrm{CH}_{4}\right)$, le protoxyde d'azote $\left(\mathrm{N}_{2} \mathrm{O}\right)$ et différents composés chlorofluorocarbonés, et par là même d'une accumulation d'énergie dans le système climatique. Ce rapport précise que la concentration de vapeur d'eau dans l'atmosphère (principal gaz à effet de serre) va également s'accroître et amplifier ce réchauffement. Les calculs de cette époque indiquent qu'en l'absence de toute action d'atténuation, le climat moyen risquerait de se réchauffer d'environ $3^{\circ} \mathrm{C}$ d'ici à la fin du XXle siècle, et le niveau de la mer de s'élever de façon significative (de 20 à $65 \mathrm{~cm}$ ).

L'alerte est clairement exprimée et, dès décembre 1989, les Nations Unies demandent aux gouvernements de mettre sur pied une Convention sur le Changement climatique. Celle-ci sera adoptée le 9 mai 1992 et sera signée par 154 pays (dont les États-Unis) lors du Sommet de la Terre de Rio de Janeiro au cours duquel s'est concrétisée la mise sur pied de deux autres conventions dédiées à la biodiversité et à la lutte contre la désertification. À travers son article 2, la Convention Climat se fixe l'objectif ultime de "stabiliser les concentrations des gaz à effet de serre à un niveau qui empêche toute perturbation anthropique dangereuse du système climatique ». C'est là un véritable défi, qui requiert de diminuer les émissions de gaz à effet de serre liées aux activités humaines.

La première COP (Conference of Parties), qui rassemble tous les pays signataires de la Convention, se réunit à Berlin en 1995. Deux ans plus tard, à Kyoto, la COP 3 aboutit à la signature d'un premier protocole visant à réduire les émissions de gaz à effet de serre des pays développés (pays dits de l'Annexe I) de 5,2 \% sur la période 2008-2012 par rapport à 1990, l'année de référence. Les décideurs politiques sont allés de l'avant, s'appuyant largement sur le diagnostic du deuxième rapport du GIEC qui conclut qu'" un ensemble d'éléments suggère une influence perceptible des activités humaines sur le climat ». Moins de dix ans se sont écoulés entre la création du GIEC et la signature de ce protocole. Mais sa mise en œuvre ne sera pas à la hauteur de l'ambition, en particu- lier parce que ce protocole n'est pas ratifié par le premier pays émetteur que sont alors les États-Unis, que préside à l'époque Georges Walker Bush. Plusieurs pays développés n'ont pas respecté leurs engagements. De plus, le mécanisme du protocole n'était pas flexible et n'était pas adapté à la croissance économique rapide de la Chine et d'autres grands pays émergents, dont les émissions de gaz à effet de serre avaient fortement augmenté.

Ces échanges entre la communauté scientifique (via les rapports du GIEC, en premier lieu) et les décideurs politiques (via les COP) se sont poursuivis depuis Kyoto. Ils s'appuient au moins en partie sur le processus d'approbation des rapports du GIEC, dont la démarche d'expertise collective garantit la qualité. Les représentants des différents pays (constituant l'assemblée plénière du GIEC) jouent un rôle décisif dans le choix des thématiques des rapports spéciaux, dans le cadrage des rapports et lors de la dernière étape, celle de l'approbation ligne à ligne de leurs résumés destinés aux décideurs. À ce stade, toute modification doit s'appuyer sur une conclusion déjà inscrite dans le rapport principal rédigé en toute liberté par les scientifiques. Ce processus d'approbation se traduit par une appropriation par les gouvernements de rapports, dont l'objectif est d'être non pas « policy prescriptive », mais « policy relevant » : le GIEC ne fait aucune recommandation, mais il fournit aux décideurs politiques les éléments pour qu'ils puissent prendre des décisions en toute connaissance de cause. Ces mêmes gouvernements s'appuient ensuite sur le diagnostic du GIEC lors des négociations conduites dans le cadre de la Convention Climat.

Mais cette dualité n'est pas sans poser problème. En 2009, juste avant la Conférence de Copenhague (COP 15), dont l'objectif était de mettre sur pied un accord « ambitieux 》 pour l'après-Kyoto, le GIEC (pourtant auréolé, en 2007, du prix Nobel de la Paix conjointement avec Al Gore) avait été l'objet d'une campagne visant à le discréditer. Cette campagne commence par le "climategate ", avec la publication d'une série de courriels piratés concernant des chercheurs britanniques de l'Université d'East Anglia, puis se renforce par la révélation d'erreurs de calcul. Les auteurs du piratage n'ont pas été identifiés. La divulgation des correspondances des scientifiques illustre, à travers des échanges d'ailleurs assez rudes, la saine divergence d'opinions qui peut exister entre des climatologues. La seule erreur avérée dans le $4^{\text {ème }}$ rapport portait sur la mesure de la fonte des glaciers himalayens. Cette erreur avait été identifiée, mais elle n'avait pu être corrigée, faute de processus le permettant ; une procédure ad hoc a été depuis mise en place. Plusieurs discours prononcés lors de

(1) Le GIEC ne réalise pas de travaux de recherche, il fait appel à des centaines de chercheurs de tous les pays pour effectuer, sur la base des publications scientifiques, une évaluation critique de l'état des connaissances en matière de changement climatique. Chaque version de ces rapports fait l'objet d'une relecture critique par des milliers de relecteurs. Enfin, leur "résumé pour décideurs » est approuvé mot par mot lors d'une session plénière des représentants de tous les pays sur la base du rapport scientifique détaillé. 
la cérémonie d'ouverture de la COP 15 de Copenhague ont fait référence à ce « climategate », ce qui a probablement rendu les négociations plus âpres et plus difficiles. La COP 15, qui a échoué à produire un nouvel accord international, a cependant marqué un tournant dans les négociations multilatérales sur le climat. Elle a inscrit l'objectif de limiter le réchauffement à moins de $2^{\circ} \mathrm{C}$ et acté le principe d'une approche basée sur des engagements volontaires de chaque pays et sur laquelle s'est construit l'Accord de Paris.

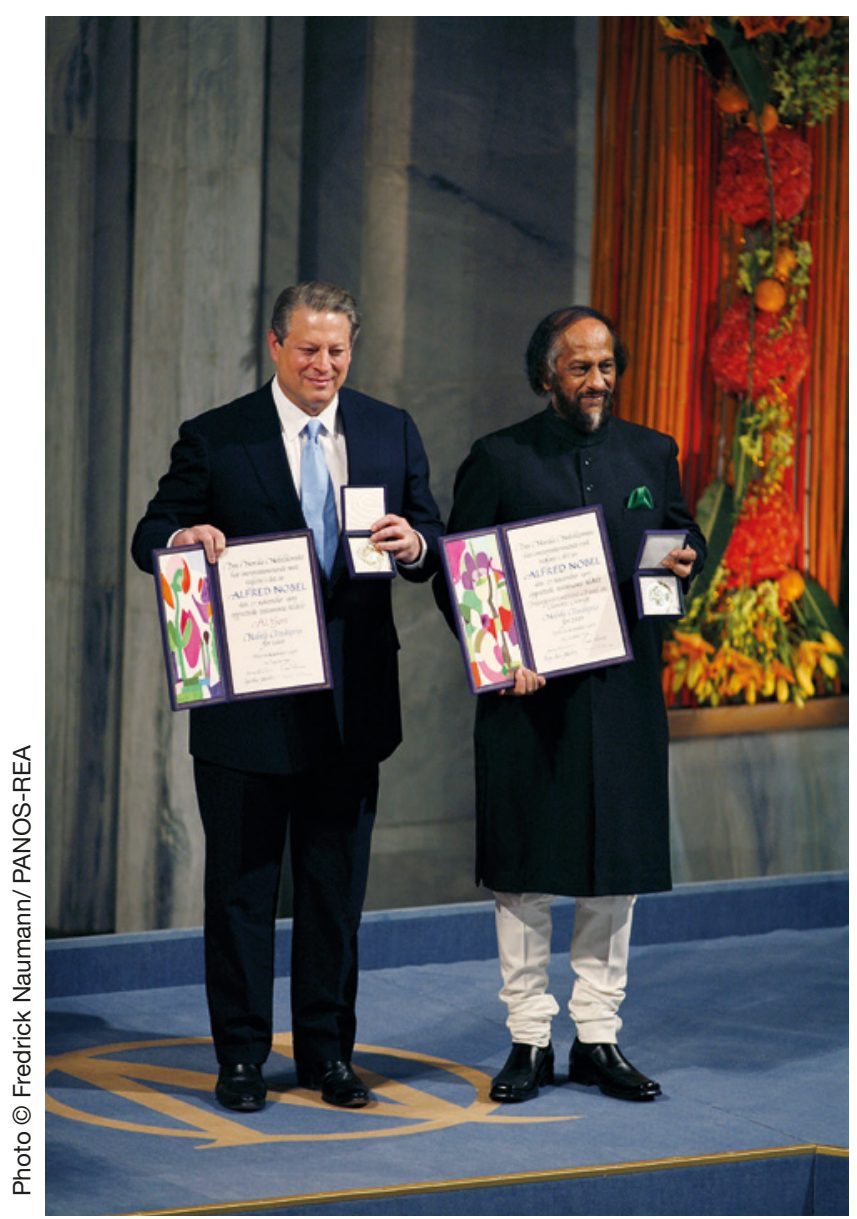

Remise du Prix Nobel de la paix conjointement à Al Gore et au Groupe intergouvernemental d'experts sur le climat (GIEC) représenté par son président, I'Indien Rajendra Pachauri, Oslo, 2007.

«En 2009, juste avant la Conférence de Copenhague (COP 15), dont l'objectif était de mettre sur pied un accord « ambitieux » pour l'après-Kyoto, le GIEC (pourtant auréolé, en 2007, du prix Nobel de la Paix conjointement avec Al Gore) avait été l'objet d'une campagne visant à le discréditer. »

En France et dans d'autres pays, cette période est marquée par la publication de livres grand public empreints d'un climato-scepticisme largement relayé dans les médias. II est tout à fait légitime que les travaux en sciences du climat et les conclusions du GIEC puissent être remis en cause par des scientifiques de toutes disciplines se-
Ion les règles du débat scientifique, en particulier dans des publications « relues par les pairs ». Néanmoins, les débats publics relèvent davantage de la polémique que d'un débat scientifique approfondi et rigoureux, avec des attaques virulentes : ainsi, le GIEC a été qualifié de «système mafieux ". Suite à ces mises en cause, une pétition signée par plus de 600 scientifiques a demandé l'ouverture d'un débat scientifique approfondi.

Organisé à l'Académie des Sciences en septembre 2010, celui-ci a confirmé la solidité des sciences du climat et des conclusions du GIEC sur les causes humaines du réchauffement climatique. Ce constat a été renforcé en 2013-2014 par les conclusions du $5^{\text {ème }}$ rapport du GIEC, qui pointe de façon quasi certaine l'influence humaine comme cause principale du réchauffement climatique des dernières décennies. Ce rapport montre également les risques d'un changement climatique non maîtrisé, les limites des capacités d'adaptation et les stratégies permettant de limiter ce réchauffement tout en assurant un développement économique. Le climato-scepticisme reste néanmoins très présent dans l'arène politique, comme en témoignent les positions adoptées par Donald Trump, le candidat républicain à la présidence des États-Unis.

La société civile n'a pas attendu les années 2000 pour s'impliquer dans le débat sur le changement climatique. En France, le Réseau Action Climat (RAC-France), affilié à l'organisation internationale Climate Action Network, fédère dès 1996 les ONG actives sur ce créneau. Certaines ONG participent au titre « d'observateurs » aux réunions du GIEC et/ou à celles de la Convention Climat. Informer, suivre les engagements, proposer des politiques publiques, dénoncer les lobbies ou les États qui freinent l'action internationale sont au cœur des actions du RAC et d'autres ONG, lesquels ont indéniablement joué un rôle clé dans la prise de conscience par les politiques, les citoyens et les entreprises de l'ampleur des problèmes posés par le réchauffement politique.

Certains secteurs industriels ont assez longtemps eu une attitude de déni qui s'est parfois traduite par un encouragement, voire un soutien à des personnalités et à des organisations susceptibles d'apporter de l'eau au moulin des climato-sceptiques, comme en témoigne l'analyse approfondie de Naomi Oreskes et Erik Conway dans leur enquête publiée sous le titre «Les marchands de doute". Ces acteurs ne se sont pas privés de tout faire pour affaiblir le diagnostic du GIEC. Très présent dans les années 1990, en particulier aux États-Unis et au Canada, cet état d'esprit s'est estompé au cours de la dernière décennie. En 2006, le rapport Stern soulignait que les conséquences du réchauffement climatique pourraient coûter de 5 à 20 fois plus cher que la mise en place d'une politique de lutte contre l'effet de serre. Même si les chiffres avancés sont contestés par certains économistes, ce rapport a indéniablement marqué un tournant dans l'attitude du monde économique vis-à-vis du réchauffement climatique.

En 2010, la conférence de Cancún (au Mexique) marque une étape importante pour tous ces acteurs non-étatiques répertoriés selon neuf catégories majeures par les Nations 


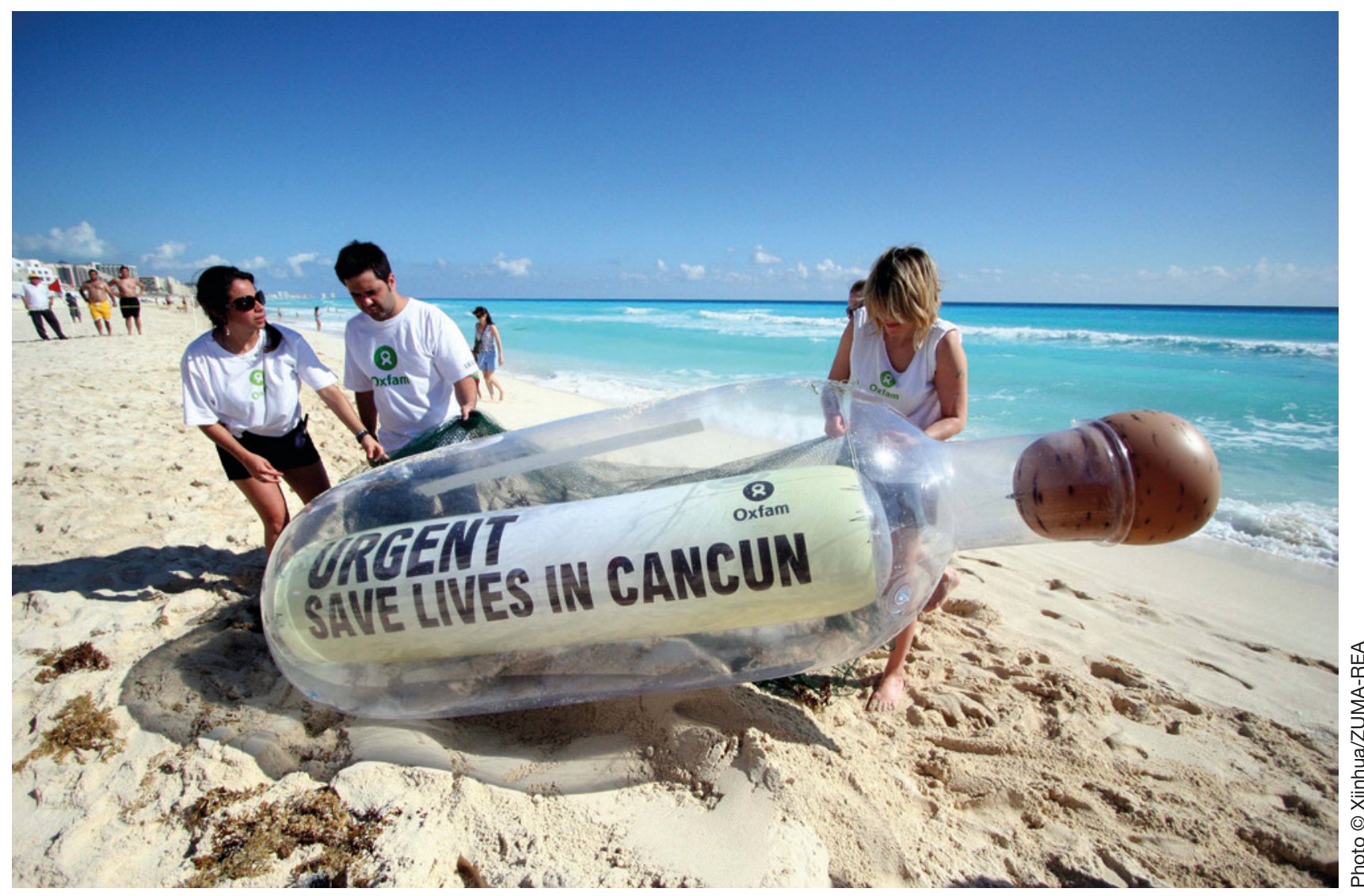

Action de communication («Une bouteille à la mer : sauvez des vies à \#Cancún ») de l'ONG Oxfam en marge de la Conférence de l'ONU sur le climat à Cancún (Mexique), novembre 2010.

«En 2010, la conférence de Cancún (au Mexique) marque une étape importante pour tous ces acteurs non-étatiques répertoriés selon neuf catégories majeures par les Nations Unies. L'accord reconnaît que "pour agir efficacement sur tous les aspects des changements climatiques, de multiples parties prenantes doivent être mobilisées aux niveaux mondial, régional, national et local, qu'il s'agisse de gouvernements, y compris les administrations infranationales et locales, d'entreprises privées ou de la société civile ..."”

Unies (2). L'accord reconnaît que " pour agir efficacement sur tous les aspects des changements climatiques, de multiples parties prenantes doivent être mobilisées aux niveaux mondial, régional, national et local, qu'il s'agisse de gouvernements, y compris les administrations infranationales et locales, d'entreprises privées ou de la société civile ... »

À côté de celui des entreprises, le rôle des collectivités - de nature assez diverse d'un pays ou d'un continent à l'autrey est mis en avant. Celles-ci n'ont pas attendu pour se mobiliser. Dès 2005, se met en place, à l'initiative du maire de Londres, le C40 qui fédère les plus grandes villes du monde ayant décidé de mettre en commun leur expérience en matière de développement durable et de lutte contre le réchauffement climatique. De même, en 2010, le Gouverneur de la Californie, Arnold Schwarzenegger, fonde le regroupement des régions mobilisées pour l'action vis-à-vis du climat, le R20, dont la mission est d'aider les gouvernements subnationaux à mettre en œuvre des projets à faibles émissions de carbone. D'autres initiatives de ce type existent : nous citerons Climate Chance, dont l'objectif est de devenir le rendez-vous régulier des acteurs non-étatiques (collectivités locales, entreprises, associations, syndicats, organismes scientifiques, citoyens) engagés dans cette lutte, avec une première réunion qui sera organisée à Nantes en septembre 2016.

Au-delà du rôle clé des gouvernements, tous ces acteurs ont contribué au succès de la Conférence de Paris, qui s'est largement appuyée sur le $5^{\text {ème }}$ rapport du GIEC. Malgré les contraintes liées à la sécurité de l'événement, les acteurs non-étatiques (ONG, entreprises et collectivités, en premier lieu) ont été très présents lors de la COP 21, que ce soit sur le site du Bourget ou dans le cadre d'évé-

(2) Les organisations non gouvernementales (ENGO), les milieux professionnels et industriels (BINGO), les administrations locales et autorités municipales (LGMA), les organisations représentant les populations autochtones (IPO), les instituts de recherche et organisations indépendantes (RINGO), les organisations syndicales (TUN$\mathrm{GO})$, les organisations de Femmes et Genre (Women \& Gender), les organisations de Jeunesse (YOUNGO) et les organisations agricoles (Farmers). 
nements comme ceux organisés au Grand Palais. La communauté scientifique s'est également engagée dans cette voie en organisant en juillet 2015 un colloque international interdisciplinaire intitulé Our common future under climate change, dont une partie importante a été consacrée au déploiement de solutions et au dialogue sciences-société. Enfin, l'année 2015 n'a pas été seulement marquée par l'Accord de Paris, elle l'a été également par la construction cohérente d'un agenda international à l'horizon 2030, qui comprend l'accord-cadre de Sendai visant à réduire les risques de catastrophes et les 17 objectifs du développement durable.

La mission d' « expertise collective », qui est celle du GIEC depuis sa création, va se poursuivre avec un $6^{\text {ème }}$ rapport, qui sera publié en 2022. D'ici là, le GIEC va également préparer trois rapports spéciaux. L'attention suscitée par les travaux du GIEC se reflète dans les attentes très fortes des différents pays qui avaient proposé 31 thématiques de rapports spéciaux ! En septembre 2018, il fournira un rapport spécial sur les impacts d'un réchauffement de $1,5^{\circ} \mathrm{C}$ au-dessus du niveau préindustriel et les trajectoires d'émissions de gaz à effet de serre associées, dans le contexte du développement durable et de l'éradication de la pauvreté, suite à l'invitation qui lui en a été faite par l'Accord de Paris. Deux autres rapports spéciaux seront rendus en 2019. Ils porteront, respectivement, sur le changement climatique, les océans et la cryosphère ${ }^{(3)}$ et sur le rapport entre le changement climatique et l'usage des sols ${ }^{(4)}$. Le GIEC renforcera l'analyse des aspects régionaux du changement climatique, ainsi que la prise en compte des impacts du changement climatique sur les villes, et les défis ainsi que les opportunités liés aux stratégies d'adaptation et d'atténuation de ces impacts.

Après l'Accord de Paris, les enjeux de recherche en sciences du climat restent multiples. Ainsi, pour la partie «physique du climat ", le Programme mondial d'étude du climat a défini 7 défis principaux correspondant aux limites des connaissances actuelles ${ }^{(5)}$. Une nouvelle initiative de recherche pour cette décennie vise à développer les connaissances permettant de répondre aux risques et aux opportunités du changement global, ainsi que les solutions de transformation vers un développement soutenable : Future Earth est une plateforme qui doit permettre de construire une recherche orientée vers les solutions susceptibles de répondre aux besoins vitaux (alimentation, eau, énergie et santé) en conciliant développement et impacts environnementaux. Pour cela, elle va renforcer la collaboration entre sciences naturelles, sciences sociales et recherche technologique afin d'identifier les meilleures solutions susceptibles d'être apportées à ces problèmes complexes. La production de ces nouvelles connaissances renforcera les rapports d'évaluation régionaux et globaux établis à l'intention des décideurs et de la société civile, qui seront associés dans une démarche de codesign et de coproduction de connaissances. Enfin, Future Earth vise également à mobiliser une nouvelle génération de scientifiques et à renforcer les capacités des pays en développement.

Le défi posé par l'Accord de Paris est immense. Respecter l'objectif des $2^{\circ} \mathrm{C}$ (et a fortiori celui de $1,5^{\circ} \mathrm{C}$ ) requiert un effort très important de recherche et d'innovation technologique et sociale. Le déploiement rapide des solutions existantes exige que les décideurs politiques et toutes les composantes de la société civile aillent rapidement de l'avant et que les citoyens du monde se sentent de plus en plus concernés par cette question de façon à préserver pour les jeunes d'aujourd'hui un climat auquel il leur sera possible de s'adapter.

(3) Zones enneigées et englacées.

(4) Désertification, dégradation des sols, gestion durable des terres, sécurité alimentaire et flux de gaz à effet de serre des écosystèmes terrestres.

(5) Mieux observer, comprendre et simuler les interactions entre nuages, circulation atmosphérique et sensibilité du climat ; évaluer la fonte des glaces et ses conséquences sur le climat ; caractériser, comprendre, attribuer et simuler les événements extrêmes (vagues de chaleur, précipitations torrentielles, tempêtes et sécheresses) en relation avec le changement climatique ; comprendre les mécanismes du changement de niveau des mers à l'échelle régionale et ses impacts sur les zones côtières ; évaluer les changements de disponibilité en eau et la sécurité d'approvisionnement; développer les prévisions climatiques à l'échelle de la décennie grâce à une meilleure compréhension des mécanismes de variabilité naturelle (comme les événements El Niño); et, enfin, mieux comprendre les processus contrôlant les puits de carbone océaniques et continentaux et leurs relations avec l'état du climat, afin d'anticiper leur évolution future. 